\title{
Impact of stochastic fluctuations in storage-discharge relations on streamflow distributions
}

\author{
S. Suweis, ${ }^{1}$ E. Bertuzzo, ${ }^{1}$ G. Botter, ${ }^{2}$ A. Porporato, ${ }^{1,3}$ I. Rodriguez-Iturbe, ${ }^{4}$ \\ and A. Rinaldo ${ }^{1,2}$ \\ Received 26 March 2009; revised 30 September 2009; accepted 5 October 2009; published 16 March 2010.
}

[1] In this paper we extend recent theoretical results on the structure of the probability density function of streamflows forced by stochastic rainfall sequences. Our extension is aimed at incorporating an additional, independent source of variability assumed to describe noisy constitutive storage-discharge relations, thought of as portraying phenomena like transient connectivity, differential activation of preferential flow paths, or macroscopic effects of spatially heterogeneous and hysteretic subsurface properties. We first show by numerical simulation that a colored noise superposed to the storage-discharge relation does not appreciably affect the overall characterization of the storage distribution. Streamflows prove more sensitive to such noise. This effect is examined by including stochasticity directly into the streamflow generation processes in the form of Gaussian multiplicative noise affecting the discharge equation of a linear reservoir. Under the above conditions, exact analytical probability distributions for the streamflow are derived. The results show that the streamflow regimes, roughly termed wet and dry to describe perennial or ephemeral streamflow regimes, are peculiarly modified by the noise and are controlled by the ratio between the subsubsurface percolation frequency, by the inverse of the mean residence time of subsurface flow, and by the noise strength. Our results suggest new and significant noise-induced phenomena, with notable ecological implications in particular for possible shifts from perennial to ephemeral regimes.

Citation: Suweis, S., E. Bertuzzo, G. Botter, A. Porporato, I. Rodriguez-Iturbe, and A. Rinaldo (2010), Impact of stochastic fluctuations in storage-discharge relations on streamflow distributions, Water Resour. Res., 46, W03517, doi:10.1029/2009WR008038.

\section{Introduction}

[2] The complete probabilistic characterization of streamflows in river basins is a primary task of hydrologic sciences because of the noteworthy implications for water resources availability and management for human needs and ecological services related, e.g., to riparian plant nutrition, preservation of fish habitat, irrigation, or storage management [e.g., Brutsaert, 2005]. Streamflows at the closure of whole river basins are the by-product of many intertwined ecohydrological and climatic processes, such as infiltration from rainfall, evapotranspiration, runoff production, and transport phenomena occurring in channeled and unchanneled regions of the basin. The intrinsic temporal variability embedded in the fluctuations of recorded runoff series thus reflects the vagaries of rainfall patterns in space and time and the random

\footnotetext{
${ }^{1}$ Laboratory of Ecohydrology, Faculté ENAC, Ecole Polytechnique Federale, Lausanne, Switzerland.

${ }^{2}$ Dipartimento di Ingegneria Idraulica, Marittima e Geotecnica, Università di Padova, Padua, Italy.

${ }^{3}$ Department of Civil and Environmental Engineering, Duke University, Durham, North Carolina, USA.

${ }^{4}$ Department of Civil and Environmental Engineering, Princeton University, Princeton, New Jersey, USA.

Copyright 2010 by the American Geophysical Union. 0043-1397/10/2009WR008038
}

character of several related hydrologic processes and landscape morphologies [e.g., Chow, 1988; Lamb and Beven, 1997; Rodriguez-Iturbe and Rinaldo, 1997; Porporato et al., 2004; Brutsaert, 2005; Eng and Milly, 2007].

[3] Stochastic fluctuations of streamflows have long been the subject of hydrologic and statistical investigations, in particular through the synthetic generation of time series obtained by deterministic models of the hydrologic response driven by stochastic climate forcings [e.g., Kottegoda and Horder, 1980]. Effective rainfall series, seen as trajectories of a stochastic process with prescribed statistical features [e.g., Xu et al., 2002] or censored through soil-water balances applied to stochastic point rainfall processes [e.g., Rodriguez-Iturbe and Porporato, 2004], have also been studied. Specific assumptions or observations are needed down to the scale of individual hydrologic processes, such as recharge due to infiltration from rainfall, losses (evapotranspiration and discharge), and storage variations related to residence time distributions in channeled and unchanneled states [e.g., Rodriguez-Iturbe and Porporato, 2004]. The temporal variability of streamflows thus reflects the stochastic nature of several underlying processes, which induces complex causal relations [e.g., Lamb and Beven, 1997; McDonnell, 1990; McGuire et al., 2005].

[4] In this general context, Botter et al. [2007a, 2007b] have recently analyzed the linkage existing between streamflow distributions and the relevant soil moisture 
dynamics in catchments. A probabilistic model of streamflow dynamics was developed therein, where the steady state probability distribution of the subsurface contribution to streamflows is analytically expressed in terms of a few macroscopic rainfall properties, soil-vegetation parameters, and key geomorphological features. The approach was initially structured in a spatially lumped framework by assuming average properties, as in the related literature on soil moisture dynamics [see, e.g., Rodriguez-Iturbe et al., 1999; Rodriguez-Iturbe and Porporato, 2004; Settin et al., 2007], and it has been later extended to tackle spatially distributed soils, vegetation, and morphological features and to derive flow duration curves [Botter et al., 2007b, 2008].

[5] Key to the exact solutions described above was the assumption of a linear, deterministic relationship between the instantaneous outflow discharge and the water volume stored in the subsurface. Such an assumption is often assumed in practice to be coupled with nonlinear net precipitation schemes [e.g., Chow, 1988; Beven, 2001] and is equivalent to assuming an invariant exponentially distributed residence time.

[6] Nonlinear storage-discharge relations have also long been considered in conceptual models of the hydrologic response (for a review, see Brutsaert [2005]). Recent studies have revamped the interest in the subject by applying the method of Brutsaert and Nieber [1977] to characterize catchments as nonlinear dynamic systems, concluding that linear storage-discharge relations are not expected in general at the scales of interest [Kirchner, 2009]. Interestingly, this applies for the class of catchments in which discharge strictly depends on the volume of water stored in the subsurface [Botter et al., 2009].

[7] However, these previous works have all considered deterministic constitutive relations between storages and streamflows, while theoretical and practical considerations, in fact, suggest that noise and variability in this constitutive relation may be an important factor [e.g., McDonnell, 1990; Beven, 2001; Berne et al., 2005; Morbidelli et al., 2006]. If runoff production schemes, for instance, account for spatial variability in the parameters characterizing the individual hydrologic processes, fluctuations inevitably emerge. For catchment-scale predictions, point-scale representations of infiltration have been challenged by numerical approaches that have accurately explored infiltration processes including runoff-run-on, rainfall variability, spatial correlation in infiltration, and rainfall fields [e.g., Beven, 2001; Berne et al., 2005; Morbidelli et al., 2006]. Moreover, connectivity patterns within a hillslope, or within any runoff-generating volume, change erratically as complex activation of preferential flow paths may result in noise in the outflow discharge [e.g., McDonnell, 1990; McGuire et al., 2005; Fiori and Russo, 2008]. Recently, Harman and Sivapalan [2009] examined how heterogeneity affects the flow response of a hillslope as a whole through numerical simulation. Overall, hydrologic storages are seen as random variables proportional to the probability of the travel times of rainfall particles to their exit boundaries [e.g., Fiori and Russo, 2008], thus sustaining our idea that random fluctuations must affect the process of runoff formation. To handle process complexity in this context, three basic approaches may be used [e.g., Guswa et al., 2002; Weiler and McDonnell, 2007; Dunn et al., 2008]: (1) work at the smallest scale and fully resolve the complex processes; (2) upscale the mathematical relationships from the small scale to develop deterministic relationships that work at the large scale; or (3) employ a stochastic approach in which the complexity is not captured explicitly but, rather, its effect is described probabilistically. We have confidently used the last approach in this paper by focusing on the key multiplicative noise effect on the storage-discharge constitutive relation.

[8] The ratio of computed instantaneous discharge and stored volume for a heterogeneous system mimicking a hillslope transport volume clearly suggests that a noisy relation is indeed the norm rather than the exception, whether or not the mean behavior of the response of the system may be approximated by a linear storage-discharge relation. Note that more than to a whole watershed, the current control volume refers to a hillslope where a local storage-discharge relation indeed makes sense. The catchment-scale complexity arises from the combined effect of serial and parallel arrangement of runoff-generating control volumes like geomorphically linked sources areas. At the chosen scale we believe that, indeed, stochastically affected hydrological processes result in greater variance of streamflows. Hydrologic connectivity [e.g., Gomi et al., 2008] also complicates matters because only a fraction of the runoff generated in a catchment actually connects with the outlet during the rainfall event. As a result, hydrologic processes are highly variable in space and time, and dynamic changes in the spatial extent and the timing of runoff-run-on phenomena call for more complex assumptions than strict determinism between subsurface storage and discharge. Moreover, it can be shown theoretically that the effects of spatial heterogeneity in material properties can be accounted for by adding a time-varying noise to the dynamic equations. Briefly, consider a system where particles are traveling along many pathways, each with a mean velocity $\bar{v}$, perturbed by a spatial random noise $\varepsilon(x)$ so that $v=\bar{v}+\varepsilon(x)$. In the limit of a large ergodic cloud of particles, we can approximate $\varepsilon(x)=$ $\varepsilon(v t) \approx \varepsilon(\bar{v} t) \propto \varepsilon(t)$ and thus incorporate the effect of the spatial variability using a time-dependent noise term (for a rigorous treatment see, e.g., Marsili et al. [1996]).

[9] The paper is organized as follows. Section 2 briefly reviews the existing theoretical background. Section 3 discusses the effects of colored noise in the storage-discharge relation. This condition seems suitable to describe many landscapes and streamflow-generating contexts. The novel mathematical model and the analytical derivation of the streamflow probability distribution are outlined in section 4 . In order to achieve exact solutions, we mimic the noise in the storage-discharge relationship by including a Gaussian multiplicative noise affecting the discharge equation of a linear reservoir. The reliability of this assumption is discussed by comparison between analytical and numerical results (section 5), with a view to noise-induced effects. A set of conclusions then closes the paper.

\section{Theoretical Framework}

[10] In this section we briefly review the modeling scheme presented by Botter et al. [2007a, 2007b], which provides a linkage between the probabilistic structure of streamflows and underlying ecohydrological, climate, and transport processes in relatively small vegetated catchments.

[11] Following Rodriguez-Iturbe and Porporato [2004] and Botter et al. [2007a], rainfall is assumed as a marked 


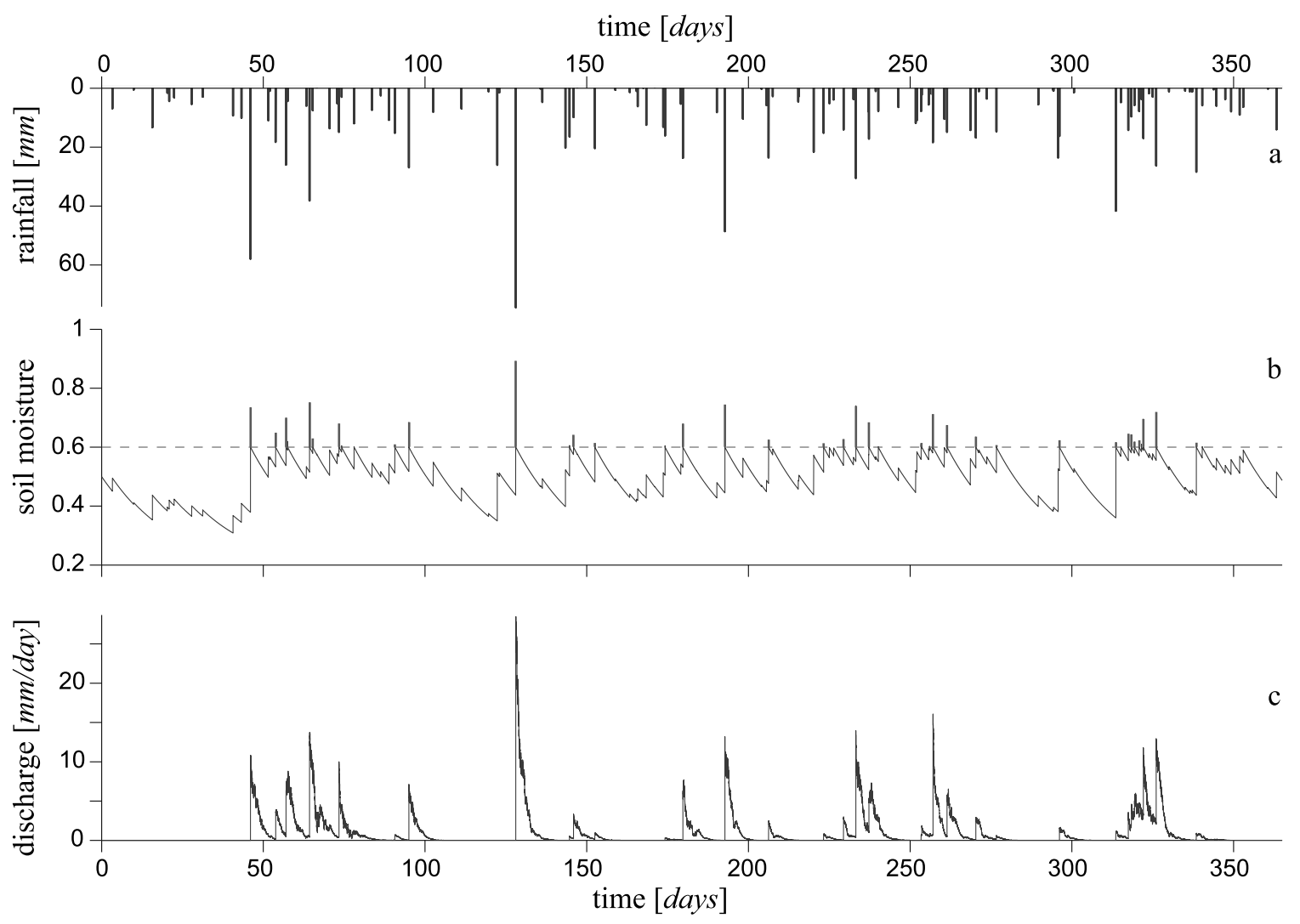

Figure 1. Schematic representation of the soil moisture and streamflow models: (a) Temporal evolution of the overall rainfall depths (simulated data). The interarrivals and the rainfall depths are exponentially distributed with a frequency $\lambda_{P}=0.3 \mathrm{~d}^{-1}$ and with normalized mean intensity $1 / \gamma_{P}=16.7 \mathrm{~mm}$, respectively. (b) Temporal evolution of the (catchment-averaged) relative soil moisture $s(t)$, which is commanded by the intermittent rainfall forcing shown in Figure 1a and by the deterministic decay due to the evapotranspiration process, according to equation (1). The dashed line represents the threshold $s_{1}$, whose up crossing determines the triggering of runoff events. The temporal sequence of rainfall excess is represented by the spikes above this threshold. (c) Temporal evolution of the overall, specific (i.e., for unit area) discharge. The soil, vegetation, and transport parameters employed for this simulations are $n=0.55$, $Z_{r}=30 \mathrm{~cm}, s_{w}=0.18, s_{1}=0.6, E T_{\max }=0.35 \mathrm{~cm} \mathrm{~d}^{-1}$, and $k=0.5 \mathrm{~d}^{-1}$.

point process with frequency $\lambda_{P}\left[T^{-1}\right]$. This assumption implicitly postulates catchment sizes (the spatial scale of our control volume in the sense of Kirchner [2009]), say, A, smaller than the spatial correlation scale of rainfall events and time scales of the process of interest greater than or equal to daily (e.g., larger than the temporal characterization of rainfall events). Furthermore, daily rainfall depths are assumed to be exponentially distributed with mean $1 / \gamma_{P}[L]$. The catchment-averaged soil moisture dynamics is modeled assuming constant (spatially and temporally averaged) soil and ecohydrological parameters: effective soil depth $Z_{r}$, porosity $n$, and maximum evapotranspiration rate $E T_{\max }$, which are assumed as representative of a prescribed season [see, e.g., Rodriguez-Iturbe et al., 1999; Rodriguez-Iturbe and Porporato, 2004]. The temporal evolution of spatially averaged relative soil moisture $s(t)$ is given by the mass balance equation within the topsoil layer of the catchment (for a review, see Rodriguez-Iturbe and Porporato [2004]):

$$
n Z_{r} \frac{d s}{d t}=-E T-L_{t}+I_{t}
$$

where $I_{t}$ represents the inflow, i.e., the stochastic increments due to infiltration from rainfall, while the losses are the evapotranspiration ET (assumed to be linear in the range of soil moisture between the wilting point $s_{w}$ and a suitable soil moisture threshold $s_{1}$ for leakage to occur) and the leakage $L_{t}$ due to deep percolation toward the deeper layer and ultimately streamflow. This model follows the minimalist probabilistic soil moisture model described in detail by Rodriguez-Iturbe and Porporato [2004]. Figures 1a and 1b show a typical realization of the stochastic rainfall model and the resulting temporal evolution of soil moisture $s(t)$.

[12] Note that the time scales chosen require focus only on subsurface contributions to streamflow, thus neglecting fast surface runoff possibly triggered by intense storms. In the absence of pronounced topographic effects and of impervious areas, however, the surface contribution to runoff is usually not significant with respect to the corresponding subsurface contribution at large time scales [e.g., McDonnell, 1990; McGuire et al., 2005; Rinaldo et al., 2006]. The latter is linked to percolation from the topsoil layer, which in turn is assumed to be triggered by the exceedance of the soil moisture threshold $s_{1}$ (Figure $1 \mathrm{~b}$ ), whose value lies typically 
between the field capacity and soil saturation. The temporal evolution of the water storage of the whole catchment can thus be schematized as the sum of the variation of the topsoil layer, described by equation (1), and that of the catchment subsurface water storage, say, $S(t)$, described by

$$
\frac{d S(t)}{d t}=-Q(t)+A \cdot L_{t}
$$

where $Q$ is the streamflow subsurface discharge and $A$ is the area of the catchment.

[13] Under the given assumptions, the spatially averaged percolation process $L_{t}$ can be well approximated by a marked Poisson processes $\xi_{t}\left(\lambda, \gamma_{P}\right)$ with frequency parameter $\lambda\left[T^{-1}\right]$ and percolation depths exponentially distributed with parameter $\gamma_{P}$. The percolation interarrival frequency can be expressed in terms of the underlying rainfall, soil, and vegetation properties as follows [Botter et al., 2007c]:

$$
\lambda=\eta \frac{\exp (-\gamma) \gamma^{\frac{\lambda_{P}}{\eta}}}{\Gamma\left(\lambda_{P} / \eta, \gamma\right)}
$$

where $\Gamma(a, b)$ is the lower incomplete gamma function of parameters $a, b, \eta=E T_{\max } /\left(n Z_{r}\left(s_{1}-s_{w}\right)\right)$ and $\gamma=\gamma_{P} n Z_{r}\left(s_{1}-s_{w}\right)$. The mass balance equation for the catchment subsurface water storage (2) can thus be expressed as

$$
\frac{d S(t)}{d t}=-Q(t)+A \xi_{t}\left(\lambda ; \gamma_{P}\right)
$$

where $\xi_{t}\left(\lambda ; \gamma_{P}\right)$ represents the time series of percolation inputs.

[14] Water pulses deeply infiltrating into soil are assumed to be released toward the stream network as subsurface or groundwater flow with a rate proportional to the instantaneous subsurface water storage, and thus, $S$ is connected to the streamflow discharge by the linear relation

$$
Q(t)=k S(t)
$$

where $k\left[T^{-1}\right]$ is the inverse of the mean residence time in subsurface. Under the above assumptions the steady state probability density function (pdf) $p(S)$ of the subsurface water storage is expressed by a Gamma distribution with shape and scale parameters $\lambda / k$ and $1 / \gamma_{P}$, respectively [Botter et al., 2007a]. By expanding the left-hand side of equation (4) as $d S / d Q \cdot d Q / d t$, a stochastic differential equation is derived for the temporal evolution of the streamflow discharge $Q(t)$ (Figure 1c):

$$
\frac{d Q(t)}{d t}=-k Q(t)+k A \xi_{t}\left(\lambda ; \gamma_{P}\right)
$$

the stationary solution of which is [Botter et al., 2007a]

$$
p(Q, t \rightarrow \infty)=p(Q) \sim Q^{\left(\frac{\lambda}{k}-1\right)} \exp \left(-\gamma_{Q} Q\right),
$$

where $\gamma_{Q}=\gamma_{P} /(k A)$ represents the mean runoff increment due to incoming percolation events.

[15] The probability distribution of $Q$ is thus related to the underlying soil and vegetation properties (through the parameter $\lambda$ ) and to key rainfall properties (through both the parameters $\gamma_{P}$ and $\lambda$ ), but it also depends on important geomorphic factors such as the mean residence time of subsurface flow $1 / k$ and the size of the basin $A$. In particular, according to equation (7), the behavior of $p(Q)$ is chiefly controlled by the ratio between the percolation frequency $\lambda$ and the inverse of the mean residence time in subsurface $k$. When $\lambda / k>1$ ("wet conditions") the pdf of the storage (runoff) is hump shaped with $p(0)=0$ (i.e., a zero storage (runoff) is characterized by zero probability), while for $\lambda / k<1$ ("dry conditions") $p(Q)$ goes to infinity for $Q \rightarrow 0$, and it monotonically decreases for $Q>0$. Although the simplifications are made, $p(Q)$ captures the observed behavior of the streamflow pdf reasonably well in many cases of practical interest [e.g., Botter et al., 2007c].

\section{Stochastic Storage-Discharge Relation}

[16] In order to take into account the stochasticity in the relation between storage and streamflow (due to transient connectivity, differential activation of preferential flow paths, and macroscopic effects of spatially heterogeneous and hysteretic subsurface properties), we add a Gaussian colored noise $l(t)$ of the Ornstein-Uhlenbeck type [Gardiner, 2004] to the linear deterministic relation described by equation (5):

$$
Q(t)=k S(t)+l(t) S(t)
$$

[17] The Ornstein-Uhlenbeck process is chosen because it is the simplest model of correlated noise. The property of nonzero correlation is physically meaningful and is specifically chosen to describe the nature of fluctuations in hydrologic processes that have nonnegligible correlation scales in both space and time. Gaussian white noise is also recovered as the zero correlation limit of the Ornstein-Uhlenbeck process. At stationarity, $l(t)$ has mean $\langle l(t)\rangle=0$ and is characterized by a correlation structure $\langle l(t) \cdot l(s)\rangle=(D \tau / 2) e^{-|t-s| / \tau}$. The relaxation time $\tau$ indicates the characteristic time for which two fluctuations cease to be correlated, while $D$ is the equivalent of a diffusion coefficient which represents the amplitude of stochastic fluctuations (note that \langle\rangle is the ensemble average operator). A colored noise, differently from white noises that have infinite variance, does not suffer from this limitation [Gardiner, 2004], and thus, $Q(t)$ in equation (8) is well defined for all $t$, ensuing to this variable an appropriate hydrologic meaning. The Ornstein-Uhlenbeck process is described by the discrete Langevin equation:

$$
l(t+\Delta t)=l(t)-\frac{1}{\tau} l(t) \Delta t+\sqrt{D} \cdot \Delta W(t),
$$

where $W$ is the Wiener process; that is, $\Delta W(t)=W(t+\Delta t)-$ $W(t)$ is a temporally uncorrelated normal random variable with mean zero and unit variance. Thus, we can study the stochastic storage-discharge relation by combining equations (4), (8), and (9). The evident hydrologic consequence of equation (8) is that now the streamflow corresponding to a given storage $S$ can fluctuate around the deterministic value $k S$ within a range that depends on the variance of the Ornstein-Uhlenbeck process.

[18] Figure 2a illustrates the typical scatter produced by the colored noise in the storage-discharge relation (obtained by suitable Monte Carlo simulation), while Figure 2b shows the results produced by the Brutsaert and Nieber [1977] method that probes the nature of the $S-Q$ relation via 

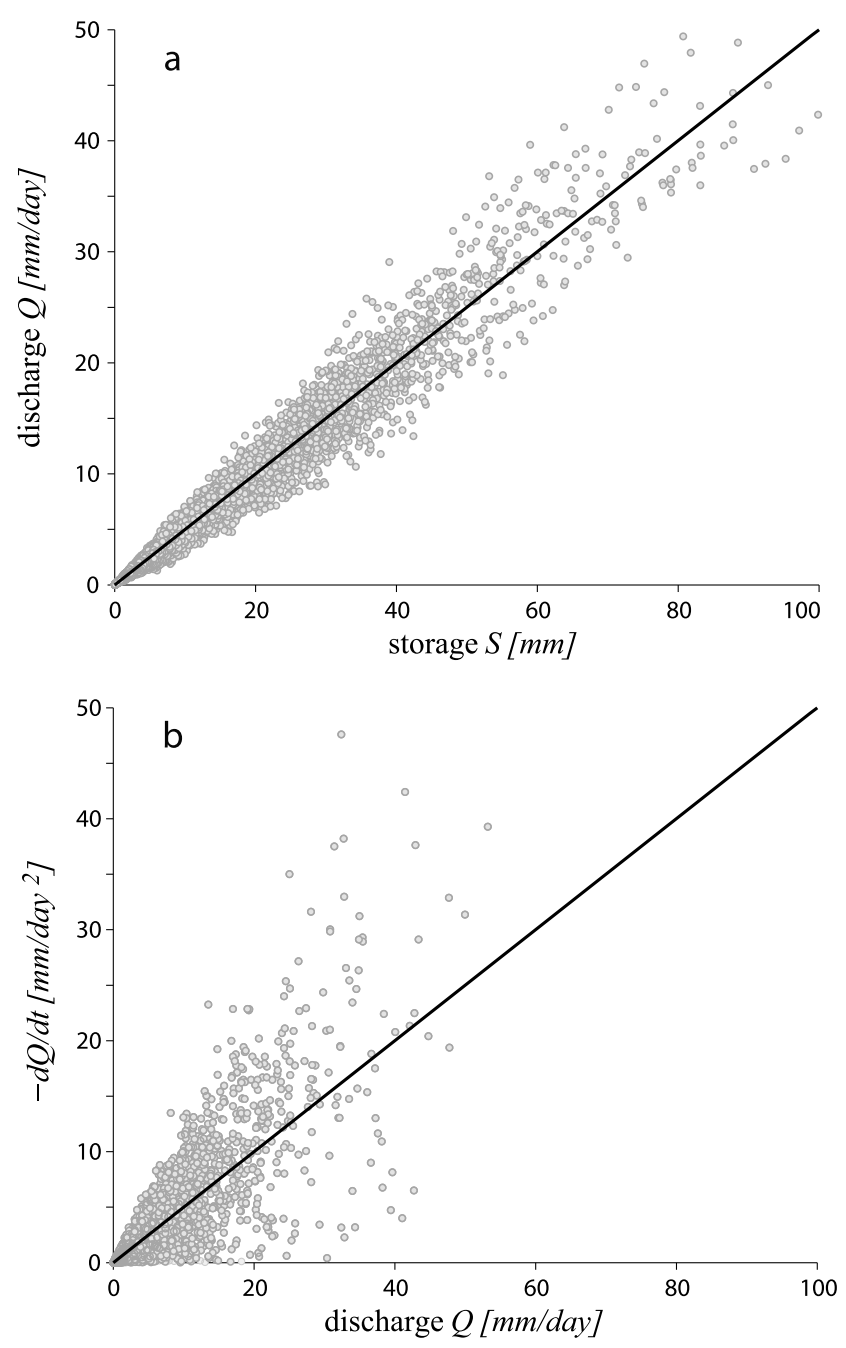

Figure 2. (a) Storage-discharge relationship arising from addition of colored noise. (b) Application of the Brutsaert and Nieber [1977] method for the $6 \mathrm{~h}$ time step that probes the nature of the $S-Q$ relation via numerical simulation of the recession curves. The parameters employed are $k=0.5 \mathrm{~d}^{-1}$ and $\operatorname{Var}(l)=0.15 k$.

numerical manipulation of the recession curves. This method can be obtained by differentiation of the axis of Figure 2a, and it allows us to estimate the mean residence time $1 / k$ solely from the measured discharge time series. Sampling effects due to the time step used in the analysis, however, enhance the noise in the $S-Q$ relation (see comparison between Figures $2 \mathrm{a}$ and $2 \mathrm{~b}$ ). It is interesting to observe that the introduction of the artificial noise qualitatively resembles field and numerically simulated data [Kirchner, 2009; Brutsaert and Nieber, 1977].

[19] Figure 3 summarizes the main results of the numerical simulation. For the integration of the Langevin equation (9) we employ the Euler-Maruyama algorithm [Kloeden and Platen, 1999]. Because negative values of the streamflow $Q$ have no physical meaning, in the simulation we impose a reflecting boundary conditions in zero for the process $(l(t)+k)$. It can be noticed that the shape of the probability distribution of the storage $p(S)$ is not significantly affected in a broad range of noise conditions (Figure 3).
On the contrary, the noise has a major effect on the streamflow distribution $p(Q)$. In particular, the mode of $p(Q)$ shifts toward zero as the strength of the noise increases. The sensitivity of $S$ and $Q$ to noise in the $Q-S$ relationship depends on hydrologic measures, such as the index of dryness $\mathcal{I}_{D}=\langle E T\rangle /\left\langle I_{t}\right\rangle$. In fact, in arid or semiarid climates where $\mathcal{I}_{D} \approx 1$, deterministic evapotranspiration drives the evolution of $S$, and thus, $p(S)$ and $p(Q)$ are less affected by intrinsic random fluctuations in the storage-discharge relation with respect to wet climates (see Figure 4). The limited effect of the noise on the $p(S)$ with respect to $p(Q)$ can be explained as follows. From equations (4) and (8), in fact, we obtain a realization of the trajectory of the storage as given by $S(t) \sim \exp \left(-\int_{0}^{t}[k+l(u)] d u\right)$. Thus, for large $t$ and for relaxation times $\tau$ short with respect to $1 / k$, the stochastic contribution to the latter integral tends to zero $(\langle l(t)\rangle=0)$. This can be generalized for any colored noise with zero mean. In fact, because of the nature of the mass balance equation, $S$ depends on the noise in an integral fashion, thus regularizing its fluctuations.

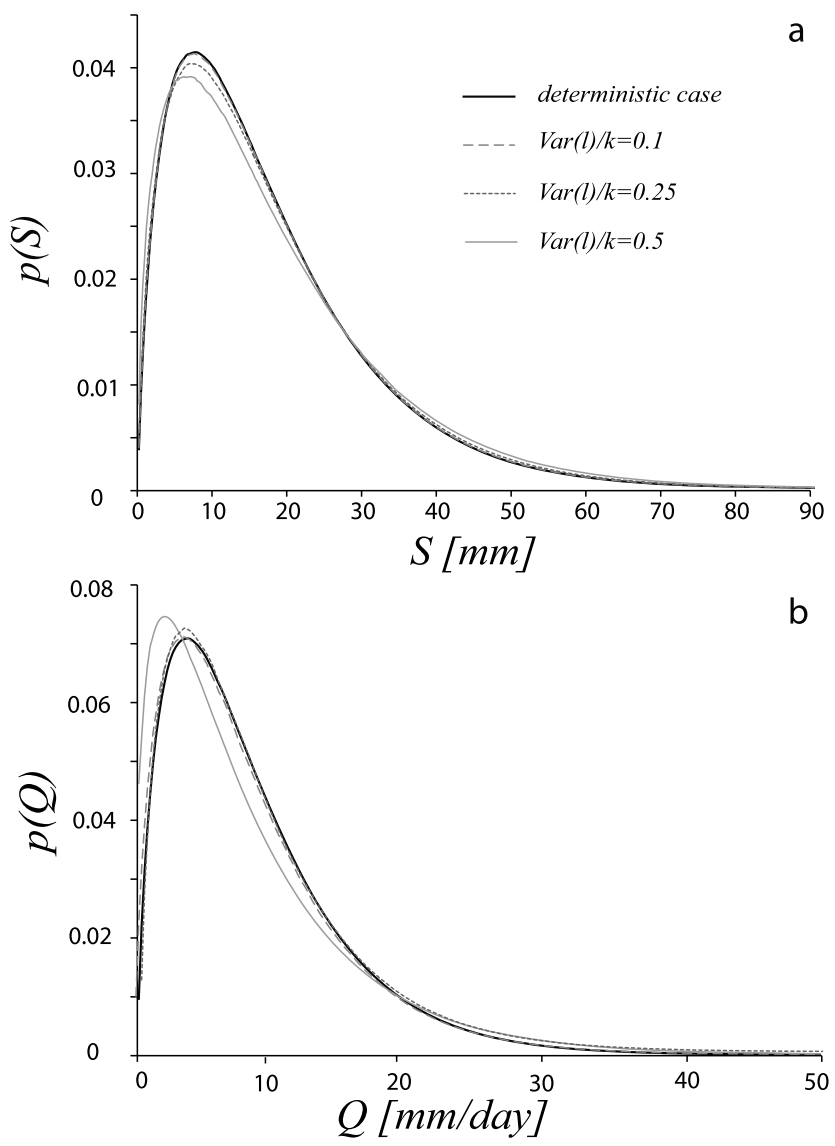

Figure 3. Probability distribution of (a) the storage $p(S)$ and (b) the streamflow $p(Q)$, calculated taking into account different variances of the colored noise in the storagestreamflow relation (equation (8)). The black curves correspond to the distributions derived from a deterministic storage-streamflow relation $Q=k S$. The parameters employed are $k=0.5 \mathrm{~d}^{-1}, \lambda=0.5 \mathrm{~d}^{-1}$, and $\tau=1 \mathrm{~d}$. The noise appreciably affects the shape of $p(Q)$, shifting the mode of the distributions toward zero even in the case of relatively low values of its variance. 

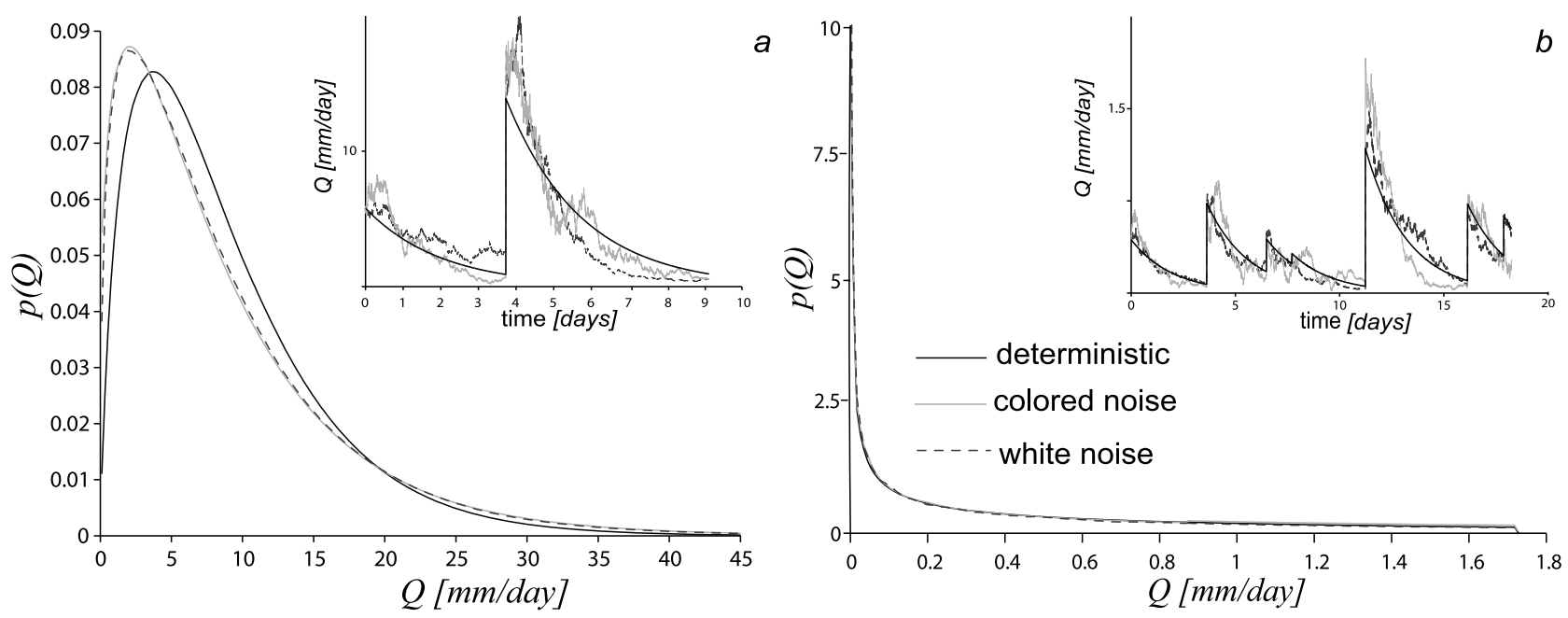

Figure 4. (a) A comparison between different numerical streamflow probability density functions $p(Q)$ for wet climate $\left(\lambda=0.875 \mathrm{~d}^{-1}, 1 / \gamma_{P}=20 \mathrm{~mm}\right.$, and $\left.k=0.5 \mathrm{~d}^{-1}\right)$. The effect of both colored and white noise is to shift the mode of the distribution toward zero. The parameters used for this calculation are $\sigma^{2}=0.125 \mathrm{~d}^{-1}$ for the white noise and $\tau=1 \mathrm{~d}, D=0.0625 \mathrm{~d}^{-1}$ for the colored noise. A numerical simulation of typical trajectories of streamflow discharge time series in a wet regime for the same cases is also shown in the inset. Note that the parameters of the white noise are tuned to reproduce the pdf of the colored noise simulation. This is deemed legitimate because we simply claim that white noise with appropriate parameters reproduce $p(Q)$ forced by colored noise. (b) The same comparisons but for dry climate $\left(\lambda=0.2 \mathrm{~d}^{-1}, 1 / \gamma_{P}=10 \mathrm{~mm}\right.$, and $\left.k=0.5 \mathrm{~d}^{-1}\right)$ and same $\sigma^{2}, \tau$, and $D$ used in Figure $4 \mathrm{a}$. It is evident how, for dry climate, $Q$ (and $S$ ) are much less affected by the intrinsic random fluctuations.

[20] The above results suggest that the noise in the storage-discharge relation significantly and interestingly influences the pdf of the streamflow. However, this framework does not allow further analytical investigation.

[21] In order to achieve exact solutions that can improve our understanding of the effect of the noise on the streamflow distribution, we mimic the stochastic fluctuation in the storage-discharge relation by including a Gaussian multiplicative noise affecting the discharge equation of a linear reservoir. The reliability of this modeling scheme is justified via numerical simulations (see Figure 4 and discussion in sections 4 and 5). In this way the stochastic relation between $S$ and $Q$ still holds, and the effect of the noise on the discharge distribution can be deepened.

\section{Noise Effects on the Streamflow Discharge Distribution}

[22] As discussed in section 3, in order to mimic the stochasticity of the streamflow generation process, we add a Gaussian white noise $\zeta(t)$ to the temporal variability of the outflow discharge equation:

$$
\frac{d Q(t)}{d t}=-k Q(t)+k A \xi_{t}\left(\lambda ; \gamma_{P}\right)+\zeta(t) Q(t),
$$

where $\langle\zeta(t)\rangle=0$ and $\langle\zeta(t) \zeta(s)\rangle=2 \sigma^{2} \delta(t-s)$; $\delta$ denotes the Dirac delta function. The physical interpretation of equation (10) is that the discharge responds to an instantaneous increase of the subsurface storage $\Delta S$ with an instantaneous increase of discharge $\Delta Q=k \Delta S$, as in the linear deterministic case, while between interstorm arrivals the linear decrease of the discharge is perturbed by the noise $\zeta(t)$.
As a result, the generalized Langevin equation (10) describes the streamflow dynamics taking into account both the variability due to the rainfall filtered by the topsoil layer and the intrinsic stochasticity of the discharge generation processes. Equation (10) is valid as long as the storage-discharge relation may be thought to hold (say, not for overland flowdominated regimes).

[23] Note that equation (10) needs be to properly interpreted by a mathematical viewpoint (see Appendix A). We find the correct mathematical interpretation by imposing the condition of the stationary storage reservoir. Taking the temporal mean of both sides of equation (4), we obtain

$$
\frac{1}{T} \int_{0}^{T} \frac{d S}{d t} d t=-\frac{1}{T} \int_{0}^{T} Q(t) d t+\frac{A}{T} \int_{0}^{T} \xi_{t}\left(\lambda ; \gamma_{P}\right) d t
$$

For $T \rightarrow \infty$ the system reaches statistical steady state, and therefore, the left-hand side of equation (11) is equal to zero and $\frac{1}{T} \int_{0}^{T} x d t \equiv\langle x\rangle$; thus, equation (11) reads as $\langle Q(t)\rangle=$ $A\left\langle\xi_{t}\left(\lambda ; \gamma_{P}\right)\right\rangle$. Analogously, from the generalized Langevin equation (10) we obtain $\langle Q(t)\rangle=A\left\langle\xi_{t}\left(\lambda ; \gamma_{P}\right)\right\rangle+\langle\zeta(t) Q(t)\rangle / k$. Therefore, the condition of the stationary reservoir translates into $\langle\zeta(t) Q(t)\rangle=0$. The latter condition is satisfied if the generalized Langevin equation is interpreted in the Itô sense [Gardiner, 2004] (Appendix A).

[24] Following the Itô interpretation, the steady state solution of equation (10) for the streamflow discharge pdf is (Appendix A)

$$
p(Q) \sim p(Q, t \rightarrow \infty)=C e^{-Q \gamma_{Q}} Q^{-\alpha+\frac{\beta}{2}-2} L_{\alpha-\frac{\beta}{2}}^{\beta}\left(Q \gamma_{Q}\right)
$$



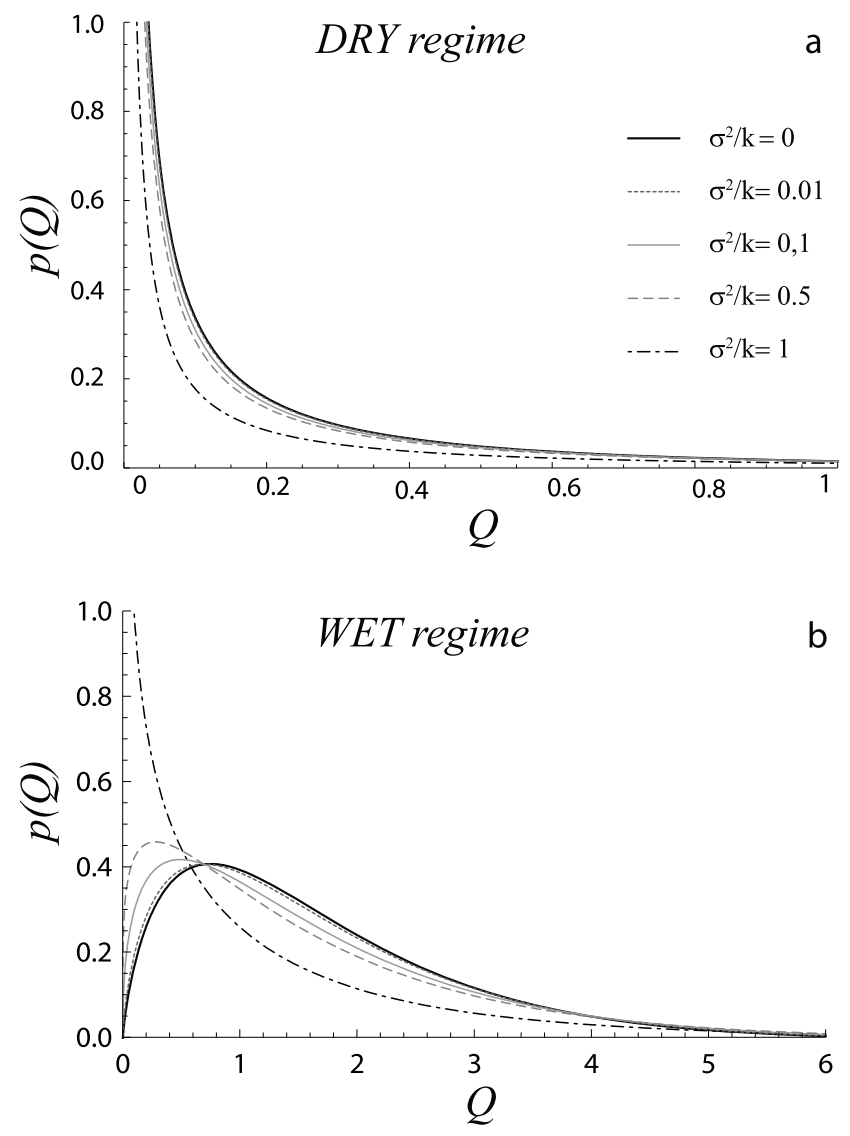

Figure 5. Analytical probability distribution of daily streamflow discharge (equation (12)) for (a) dry regime $(\lambda / k=0.04)$ and (b) wet regime $(\lambda / k=1.75)$. Different lines represent different values of the dimensionless parameter ratio $\sigma^{2} / k$. Note that $\sigma^{2} / k=0$ refers to the case of the deterministic reservoir (equation (7)).

where $C$ is the normalization constant, $\alpha=k / 2 \sigma^{2}-1 / 2$, $\beta=\sqrt{4 \lambda \sigma^{2}+\left(\sigma^{2}+k\right)^{2}} / \sigma^{2}$, and $L_{a}^{b}(x)$ is the generalized Laguerre polynomial [Abramowitz and Stegun, 1965]. A remarkable property of $p(Q)$ is that it has a power law tail, i.e., for $Q \rightarrow \infty$

$$
p(Q) \propto Q^{-\left(2+k / \sigma^{2}\right)}
$$

and thus, just adding a multiplicative Gaussian noise to the linear decay of the discharge, the tail of the streamflow distribution changes from the exponential (equation (7)) to the power law (equation (13)). This also induces a change in the mode of the distribution, thus substantially altering the streamflow regimes with respect to the purely deterministic linear case.

\section{Results and Discussion}

[25] A comparison between numerical streamflow probability density functions corresponding to three different cases (deterministic and white and colored noise) is shown in Figure 4. While for wet cases (Figure 4a) both colored and white noise produce the effect of shifting the mode of the distribution toward zero, in dry climates (Figure $4 \mathrm{~b}) Q$ (and $S$ ) are much less affected by the noise. The results shown in Figure 4 support the assumption of considering a noisy storage-discharge relation as a byproduct of a memoryless stochasticity in the streamflow generation processes. A numerical simulation of typical trajectories of streamflow discharge time series for the same cases is also reported in the inset of Figure 4.

[26] Figure 5 shows the effects induced by the white multiplicative noise on a dry streamflow distribution (equation (12)). As the noise $\left(\sigma^{2} / k\right)$ increases, the probability of observing low values of streamflow increases, thus increasing the dryness on the streamflow regime. Notice, however, that $\langle Q\rangle$ remains constant. That is, $\langle Q\rangle$ does not depend on $\sigma^{2}$; that is, $\langle Q\rangle=\left\langle\xi_{t}\left(\lambda, \gamma_{P}\right)\right\rangle$. The higher probabilities for low $Q$ are indeed balanced by increased probabilities of high discharge owing to their algebraic decay (see equation (13)). In other terms, the presence of the noise does not modify the mean of the streamflow pdf, but it significantly increases its variance (see Figure 6).

[27] The noise induces similar effects on the wet streamflow distribution as shown in Figure 5b. As the noise strengthens, an increased probability of high discharge is observed, and it is balanced by a shift of the mode of $p(Q)$ toward zero. Interestingly, above a certain threshold of the noise, a shift from wet to dry regime occurs. According to equation (12) the shift between the two different regimes is controlled by the exponent of the power in equation (12). The function $f\left(\lambda / k, \sigma^{2} / k\right)=3 \sigma^{2}+k-$ $\sqrt{\sigma^{4}+2 k \sigma^{2}+4 \lambda \sigma^{2}+k^{2}} / 2 \sigma^{2}$ of the two dimensionless parameters ratio $\lambda / k$ and $\sigma^{2} / k$ defines such shift. When $f$ $\left(\lambda / k, \sigma^{2} / k\right)<0$, the pdf of the runoff is hump shaped with $p(Q=0)=0$ (wet regime), while for $f\left(\lambda / k, \sigma^{2} / k\right)>0$ (dry regime) $p(Q)$ goes to infinity for $Q \rightarrow 0$, and it monotonically decreases for $Q>0$. The threshold between the two regimes is

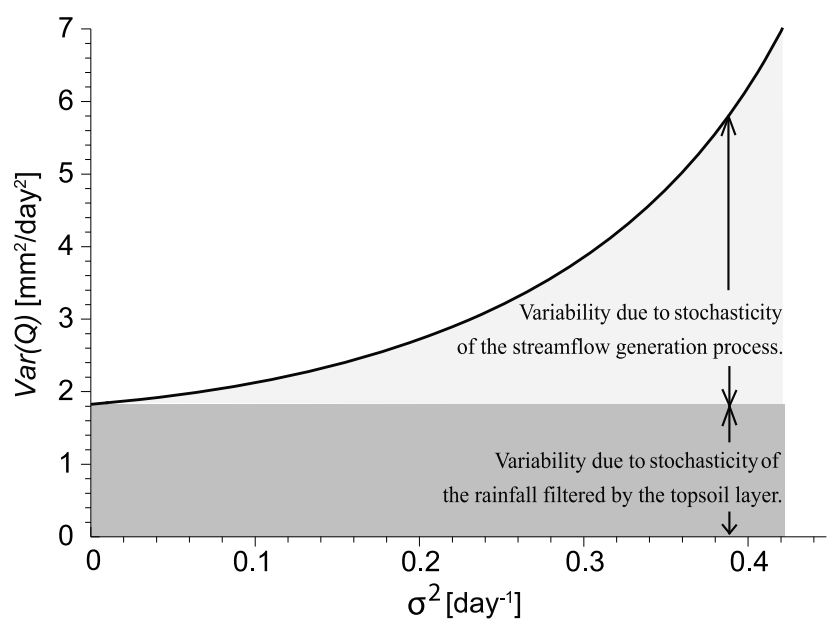

Figure 6. Numerical calculation of the variance $\operatorname{Var}(Q)=$ $\left\langle Q^{2}\right\rangle-\langle Q\rangle^{2}$ of the streamflow discharge pdf (for parameters $\lambda=0.875 \mathrm{~d}^{-1}$ and $\left.k=0.5 \mathrm{~d}^{-1}\right)$ as a function of the intensity of the white noise $\sigma^{2}$. For $\sigma^{2}=0$ we exactly obtain the variance of the Gamma distribution in equation (7) with the same parameters $\lambda$ and $k$ that refer to the deterministic linear reservoir. The different contributions to $\operatorname{Var}(Q)$ are shown by light gray and dark gray. 


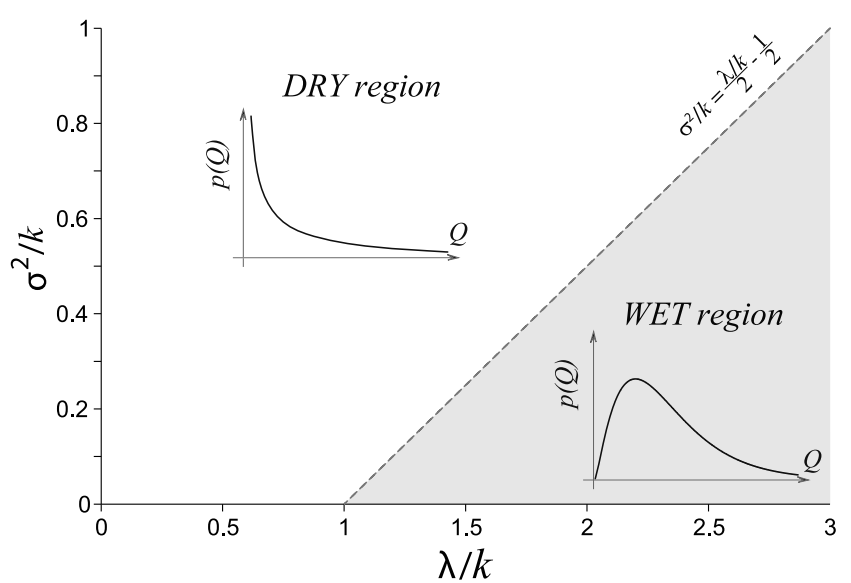

Figure 7. Representation of the streamflow discharge distribution regime as a function of the dimensionless parameters $\lambda / k$ and $\sigma^{2} / k$. Notice that the noise induces a shift from wet to dry regime.

derived by imposing $f\left(\lambda / k, \sigma^{2} / k\right)=0$, from which one finds (see Figure 7)

$$
\frac{\sigma^{2}}{k}=\frac{\lambda}{2 k}-\frac{1}{2}
$$

[28] Therefore, taking a given value $\lambda k$ that would correspond to a wet regime when $\sigma^{2}=0$ may now correspond to a dry regime if $\sigma^{2}$ is above $\lambda / 2-k / 2$. This noise-induced shift, which is similar to those noted in other contexts [e.g., Horsthemke and Lefever, 1983], occurs also for colored noise, as we have verified numerically.

[29] Notice that in the framework described in section 4, for each realization of $Q(t)$ it is possible to obtain the corresponding time series of $S$ by integration of equation (4). We underline the fact that, for a given time window, $\Delta Q / \Delta S$ is still free to fluctuate around its corresponding deterministic value $k$.

[30] Underestimating hydrologic variability may have ecological consequences. Noise-induced wet-dry transitions (thought of as reproducing regime shifts from perennial to ephemeral streamflows) are important because low-stage discharges are crucial to establish natural flow conditions for the preservation of fish and riparian habitats. Note, in particular, that minimum flows are the most used targets in water resources management to sustain aquatic ecosystems.

\section{Conclusions}

[31] The following conclusions are worth emphasizing.

[32] 1. Numerical simulation has shown that a physically meaningful colored noise in the storage-discharge relation influences appreciably the discharge distribution. In particular, a shift of the mode of the pdf toward zero is observed. We assumed that the noise in the storage-discharge relation may be surrogated by considering stochasticity directly in the streamflow generation processes. In such a case we can analytically derive the probability distribution of streamflows, whose properties reflect the results obtained by numerical simulation employing colored noise. The theoretical framework couples a stochastic description of soil moisture dynamics with a transport model that embeds the variability of the streamflow generation process through a multiplicative Gaussian noise.

[33] 2. The effect of the multiplicative Gaussian noise on the streamflow distribution is significant. In particular, the tail of the streamflow distribution $p(Q)$ changes from exponential to a power law type with heavy tail. As the noise increases, the probability of observing low values of streamflow also increases, and the mode of $p(Q)$ shifts toward zero. Higher probabilities for low $Q$ are balanced by an increased probability of high discharge; that is, the power law tail becomes fatter. Therefore, the presence of the noise does not change the mean of $Q$ but significantly increases its variance. Thus, neglecting these additional environmental fluctuations may produce underestimations of the variability of streamflows, with relevant ecohydrological consequences.

[34] 3. Above a threshold in the noise magnitude, a shift from wet to dry regime occurs, implying a major ecological impact owing to a change from perennial to ephemeral streamflows. The transition between the two different regimes is controlled by parameters of clear physical meaning, and we propose an analytical expression for the related threshold.

\section{Appendix A: Analytical Solution of the Generalized Langevin Equation}

[35] In this section we provide the derivation of the analytical solution of the generalized Langevin equation

$$
\frac{d Q(t)}{d t}=-k Q(t)+\zeta(t) Q(t)+k A \xi_{t}\left(\lambda ; \gamma_{P}\right),
$$

where $\zeta(t)$ is a Gaussian white noise with mean, $\langle\zeta(t)\rangle=0$, correlation $\langle\zeta(t) \zeta(s)\rangle=2 \sigma^{2} \delta(t-s)$, and

$$
\xi_{t}\left(\lambda ; \gamma_{P}\right)=\frac{1}{k A} \sum_{i=1}^{N(t)} \Delta Q_{i} \delta\left(t-t_{i}\right)
$$

is a compound Poisson process [Snyder, 1979], where $\{N(t)$, $t \geq 0\}$ is an homogeneous Poisson counting process of rate $\lambda$ and $\left\{Q_{i}\right\}$ is a sequence of mutually independent $N(t)$ and is also independent of $N(t)$, identically distributed random variables with a probability density function $b(Q)$. In our particular case $b(Q)$ is an exponential distribution with parameter $\gamma_{Q}=\gamma_{P} / A k$. From a mathematical point of view equation (A1), as it stands, is meaningless [Gardiner, 2004; Van Kampen, 2007]. In fact, according to equation (A1), each pulse in $\zeta(t)$ gives rise to a pulse in $Q$ and hence a jump in $Q$. Thus, the value of $Q$ in the right-hand side of equation (A1) is undetermined. The incremental form of equation (A1) is

$$
d Q(t)=-k Q(t) d t+d W(t) Q(t)+k A \xi_{t}\left(\lambda ; \gamma_{P}\right) d t,
$$

where $W(t)=\int_{0}^{t} \zeta\left(t^{\prime}\right) d t^{\prime}$ is the well-known Wiener process. The solution of (A3) can be written in the integral form

$$
\begin{aligned}
Q(t)= & Q\left(t_{0}\right)-k \int_{t_{0}}^{t} Q\left(t^{\prime}\right) d t^{\prime}-\int_{t_{0}}^{t} d W\left(t^{\prime}\right) Q\left(t^{\prime}\right) \\
& +\int_{t_{0}}^{t} \xi_{t^{\prime}}(\lambda ; \gamma) d t^{\prime}
\end{aligned}
$$


The second integral in the right-hand side of equation (A4) is a stochastic integral, and it is defined as the limit of the partial sum [Gardiner, 2004]

$$
S_{n}=\sum_{i=1}^{n} Q\left(\tau_{i}\right)\left[W\left(t_{i}\right)-W\left(t_{i-1}\right)\right]
$$

where $t_{0} \leq t_{1} \leq . . \leq t_{n-1} \leq t$ and $t_{i-1} \leq \tau_{i} \leq t_{i}$. It can be shown that $S_{n}$ depends on the particular choice of $\tau_{i}$ [Gardiner, 2004], and thus, its value depends on the particular interpretation chosen for (A5). One of the most famous interpretations is that of Itô [Ito, 1951]:

$$
S_{n}(\text { Itô })=\sum_{i=1}^{n} Q\left(\tau_{i}=t_{i-1}\right)\left[W\left(t_{i}\right)-W\left(t_{i-1}\right)\right]
$$

[36] We underline the fact that different interpretations lead to different results, so the correct and coherent interpretation of equations (A1), (A3), and (A4) is crucial for both analytical and computational calculations. In particular, we emphasize that if equation (A1) is interpreted in the Itô sense, then $\langle Q(t) \zeta(t)\rangle_{\text {Ito }}=\langle Q(t)\rangle\langle\zeta(t)\rangle=0$. The former property straightforwardly derives from the observation that in the Itô interpretation $Q(t)$ at time $t$ does not depend on the noise $\zeta(t)$ at the same time.

[37] Accordingly, equation (A1) corresponds to the forward Chapman-Kolmogorov equation [Gardiner, 2004; Van Kampen, 2007]

$$
\begin{aligned}
\frac{\partial}{\partial Q} p(Q, t)= & k \frac{\partial}{\partial Q}[Q \cdot p(Q, t)]+\sigma^{2} \frac{\partial^{2}}{\partial Q^{2}}\left[Q^{2} p(Q, t)\right]-\lambda p(Q, t) \\
& +\lambda \int_{0}^{Q} \gamma_{Q} e^{-\gamma_{Q}(Q-z)} p(z, t) d z
\end{aligned}
$$

which in turn can be written in the form of a continuity equation

$$
\frac{\partial}{\partial Q} p(Q, t)=-\frac{\partial}{\partial Q} J(Q, t)
$$

where the probability current $J(Q, t)$ is [Daly and Porporato, 2006]

$J(Q, t)=-k p(Q, t)-\sigma^{2} \frac{\partial}{\partial Q}\left[Q^{2} p(Q, t)\right]+\lambda \int_{0}^{Q} e^{-\gamma_{Q}(Q-z)} p(z) d z$.

[38] Imposing a natural boundary, the probability current vanishes in steady state conditions, so we have that the steady state solution $p(Q, t \rightarrow \infty)$ satisfies $J(Q)=0 \forall Q$. Multiplying both sides of the former equation by $e^{Q_{\gamma_{Q}}}$ and differentiating with respect to $Q$, one gets the steady state equation

$$
\begin{gathered}
Q^{2} p^{\prime \prime}(Q)+\left(\left(\frac{k}{\sigma^{2}}+4\right) Q+\gamma_{Q} Q^{2}\right) p^{\prime}(Q)+\left(\left(\frac{k \gamma_{Q}}{\sigma^{2}}+2 \gamma_{Q}\right) Q\right. \\
\left.+\left(2+\frac{k}{\sigma^{2}}-\frac{\lambda}{\sigma^{2}}\right)\right) p(Q)=0 .
\end{gathered}
$$

[39] The solution of equation (A10) is [Polyanin and Zaitsev, 2003]

$$
\begin{aligned}
p(Q)= & e^{-Q \gamma_{Q}} Q^{-\alpha+\frac{\beta}{2}-2}\left(C_{1} U\left[\frac{\beta}{2}-\alpha, \beta+1, Q \gamma_{Q}\right]\right. \\
& \left.+C_{2} L_{\alpha-\frac{\beta}{2}}^{\beta}\left[Q \gamma_{Q}\right]\right),
\end{aligned}
$$

where $\alpha=\frac{k}{2 \sigma^{2}}-\frac{1}{2} ; \beta=\frac{\sqrt{4 \lambda \sigma^{2}+\left(\sigma^{2}+k\right)^{2}}}{\sigma^{2}} ; C_{1}, C_{2}$ are integration constants; and $U[a, b, z]$ and $L_{n}^{\nu}[z]$ are the confluent hypergeometric function and the generalized Laguerre polynomial, respectively [Abramowitz and Stegun, 1965]. For $C_{1} \neq 0$ all moments of $p(Q)$ are infinite; then for physical reasons we take $C_{1}=0$. Thus, the appropriate solution of (A1) is

$$
p(Q)=C_{2} e^{-Q \gamma_{Q}} Q^{-\alpha+\frac{\beta}{2}-2} L_{\alpha-\frac{\beta}{2}}^{\beta}\left[Q \gamma_{Q}\right]
$$

where $C_{2}$ can be determined by the normalization condition $\int_{0}^{\infty} p(Q) d Q=1$. Finally, notice that in the limit $\sigma^{2} \rightarrow 0$ the Chapman-Kolmogorov equation (A7) becomes

$$
\begin{aligned}
\frac{\partial}{\partial Q} p(Q, t)= & k \frac{\partial}{\partial Q}[Q \cdot p(Q, t)]-\lambda p(Q, t) \\
& +\lambda \int_{0}^{Q} \gamma_{Q} e^{-\gamma_{Q}(Q-z)} p(z, t) d z
\end{aligned}
$$

which corresponds exactly to the equation for the streamflow pdf previously derived [see Botter et al., 2007a] and with state solution $p(Q) \sim Q^{\left(\frac{\lambda}{k}-1\right)} \exp \left(-\gamma_{Q} Q\right)$.

[40] Acknowledgments. This research is supported by funds provided by the ERC Advanced Grant RINEC-227612. Funds provided by the Faculté ENAC, Ecole Polytechnique Fédérale of Lausanne are also acknowledged. A.-P. acknowledges the visiting Landolt Chair program for financial support, and I.R.-I. acknowledges the support of the U.S. National Science Foundation under grant EAR-0642550. The writers wish to thank Andrew J. Guswa and two anonymous reviewers for insightful comments on a draft of the manuscript.

\section{References}

Abramowitz, M., and I. Stegun (1965), Handbook of Mathematical Functions, Dover, New York.

Berne, A., R. Uijlenhoet, and P. A. Troch (2005), Similarity analysis of subsurface flow response of hillslopes with complex geometry, Water Resour. Res., 41, W09410, doi:10.1029/2004WR003629.

Beven, K. (2001), Rainfall-Runoff Modeling: The Primer, John Wiley, Chichester, U. K.

Botter, G., A. Porporato, I. Rodriguez-Iturbe, and A. Rinaldo (2007a), Basin-scale soil moisture dynamics and the probabilistic characterization of carrier hydrologic flows: Slow, leaching-prone components of the hydrologic response, Water Resour. Res., 43, W02417, doi:10.1029/ 2006WR005043.

Botter, G., A. Porporato, E. Daly, I. Rodriguez-Iturbe, and A. Rinaldo (2007b), Probabilistic characterization of base flows in river basins: Roles of soil, vegetation, and geomorphology, Water Resour. Res., 43, W06404, doi:10.1029/2006WR005397.

Botter, G., F. Peratoner, A. Porporato, I. Rodriguez-Iturbe, and A. Rinaldo (2007c), Signatures of large-scale soil moisture dynamics on streamflow statistics across U.S. climate regimes, Water Resour. Res., 43, W11413, doi:10.1029/2007WR006162.

Botter, G., E. Daly, A. Porporato, I. Rodriguez-Iturbe, and A. Rinaldo (2008), Probabilistic dynamics of soil nitrate: Coupling of ecohydrological and biogeochemical processes, Water Resour. Res., 44, W03416, doi:10.1029/2007WR006108.

Botter, G., A. Porporato, I. Rodriguez-Iturbe, and A. Rinaldo (2009), Nonlinear storage-discharge relations and catchment streamflow regimes, Water Resour. Res., 45, W10427, doi:10.1029/2008WR007658.

Brutsaert, W. (2005), Hydrology: An Introduction, Cambridge Univ. Press, New York. 
Brutsaert, W., and J. L. Nieber (1977), Regionalized drought flow hydrographs from a mature glaciated plateau, Water Resour. Res., 13(3), 637-643, doi:10.1029/WR013i003p00637.

Chow, V. (1988), Applied Hydrology, McGraw-Hill, New York.

Daly, E., and A. Porporato (2006), Probabilistic dynamics of some jump-diffusion systems, Phys. Rev. E, 73(2), 026108, doi:10.1103/ PhysRevE.73.026108.

Dunn, S., J. Freer, and M. E. A. Weiler (2008), Conceptualization in catchment modelling: Simply learning?, Hydrol. Processes, 22(13), 2389-2393.

Eng, K., and P. C. D. Milly (2007), Relating low-flow characteristics to the base flow recession time constant at partial record stream gauges, Water Resour. Res., 43, W01201, doi:10.1029/2006WR005293.

Fiori, A., and D. Russo (2008), Travel time distribution in a hillslope: Insight from numerical simulations, Water Resour. Res., 44, W12426, doi:10.1029/2008WR007135.

Gardiner, C. W. (2004), Handbook of Stochastic Methods for Physics, Chemistry, and the Natural Sciences, 3rd ed., Springer, Berlin.

Gomi, T., R. C. Sidle, S. Miyata, K. Kosugi, and Y. Onda (2008), Dynamic runoff connectivity of overland flow on steep forested hillslopes: Scale effects and runoff transfer, Water Resour. Res., 44, W08411, doi:10.1029/2007WR005894.

Guswa, A. J., M. A. Celia, and I. Rodriguez-Iturbe (2002), Models of soil moisture dynamics in ecohydrology: A comparative study, Water Resour. Res., 38(9), 1166, doi:10.1029/2001WR000826.

Harman, C., and M. Sivapalan (2009), Effects of hydraulic conductivity variability on hillslope-scale shallow subsurface flow response and storage-discharge relations, Water Resour. Res., 45, W01421, doi:10.1029/ 2008WR007228.

Horsthemke, W., and R. Lefever (1983), Noise-Induced Transitions, Springer, Berlin.

Ito, K. (1951), Multiple Wiener integral, J. Math. Soc. Jpn., 3, 157-169.

Kirchner, J. W. (2009), Catchments as simple dynamical systems: Catchment characterization, rainfall-runoff modeling, and doing hydrology backward, Water Resour. Res., 45, W02429, doi:10.1029/2008WR006912.

Kloeden, P., and E. Platen (1999), Numerical Solution of Differential Stochastic Equations, Springer, Berlin.

Kottegoda, N., and M. Horder (1980), Daily flow model based on rainfall occurences using pulses and a transfer function, J. Hydrol., 47, 215-234.

Lamb, R., and K. Beven (1997), Using interactive recession curve analysis to specify a general catchment storage model, Hydrol. Earth Syst. Sci., 1, 101-113.

Marsili, M., A. Maritan, F. Toigo, and J. R. Banavar (1996), Stochastic growth equations and reparametrization invariance, Rev. Mod. Phys., 68(4), 963-983, doi:10.1103/RevModPhys.68.963.

McDonnell, J. J. (1990), A rationale for old water discharge through macropores in a steep, humid catchment, Water Resour. Res., 26(11), 2821-2832, doi:10.1029/WR026i011p02821.
McGuire, K. J., J. J. McDonnell, M. Weiler, C. Kendall, B. L. McGlynn, J. M. Welker, and J. Seibert (2005), The role of topography on catchmentscale water residence time, Water Resour. Res., 41, W05002, doi:10.1029/ 2004WR003657.

Morbidelli, R., C. Corradini, and R. Govindaraju (2006), A field-scale infiltration model accounting for spatial heterogeneity of rainfall and soil saturated hydraulic conductivity, Hydrol. Processes, 20(7), 1465-1481, doi:10.1002/hyp.5943.

Polyanin, A., and V. Zaitsev (2003), Handbook of Exact Solutions for Ordinary Differential Equations, 2nd ed., Chapman and Hall, Boca Raton, Fla.

Porporato, A., E. Daly, and I. Rodriguez-Iturbe (2004), Soil water balance and ecosystem response to climate change, Am. Nat., 164(5), 625-632.

Rinaldo, A., G. Botter, E. Bertuzzo, A. Uccelli, T. Settin, and M. Marani (2006), Transport at basin scales: 1. Theoretical framework, Hydrol. Earth Syst. Sci., 10(1), 19-29.

Rodriguez-Iturbe, I., and A. Porporato (2004), Ecohydrology of Water Controlled Ecosystems: Soil Moisture and Plant Dynamics, Cambridge Univ. Press, New York.

Rodriguez-Iturbe, I., and A. Rinaldo (1997), Fractal River Basins: Chance and Self-Organization, Cambridge Univ. Press, New York.

Rodriguez-Iturbe, I., A. Porporato, L. Ridolfi, V. Isham, and D. R. Cox (1999), Probabilistic modelling of water balance at a point: The role of climate, soil and vegetation, Proc. Soc London, R., Ser. A, 455, 3789-3805.

Settin, T., G. Botter, I. Rodriguez-Iturbe, and A. Rinaldo (2007), Numerical studies on soil moisture distributions in heterogeneous catchments, Water Resour. Res., 43, W05425, doi:10.1029/2006WR005737.

Snyder, D. (1979), Random Point Processes, John Wiley, New York.

Van Kampen, N. G. (2007), Stochastic Processes in Physics and Chemistry, 3rd ed., North Holland, Amsterdam.

Weiler, M., and J. J. McDonnell (2007), Conceptualizing lateral preferential flow and flow networks and simulating the effects on gauged and ungauged hillslopes, Water Resour. Res., 43, W03403, doi:10.1029/ 2006WR004867.

Xu, Z., G. Schultz, and A. Schumann (2002), DA conceptually-based stochastic point process model for daily stream-flow generation, J. Hydrol., 16, 3003-3017.

E. Bertuzzo, A. Rinaldo, and S. Suweis, Laboratory of Ecohydrology, Faculté ENAC, Ecole Polytechnique Federale, CH-1015 Lausanne, Switzerland.

G. Botter, Dipartimento di Ingegneria Idraulica, Marittima e Geotecnica, Università di Padova, I-35131 Padova, Italy.

A. Porporato, Department of Civil and Environmental Engineering, Duke University, Durham, NC 27708-0287, USA.

I. Rodriguez-Iturbe, Department of Civil and Environmental Engineering, Princeton University, Princeton, NJ 08544, USA. 